\title{
The effects of mobile health on emergency care in low- and middle-income countries: A systematic review and narrative synthesis
}

W Tyler Winders ${ }^{1}$,

Stephanie C Garbern², Corey B Bills ${ }^{3}$, Pryanka Relan ${ }^{4}$, Megan L Schultz ${ }^{5}$, Indi Trehan ${ }^{6}$, Sean M Kivlehan, Torben K Becker ${ }^{8 *}$, Ruth McQuillan9*; on behalf of GEMLR

\footnotetext{
${ }^{1}$ School of Public Health, University of Edinburgh, Edinburgh, UK

${ }^{2}$ Department of Emergency Medicine, Alpert Medical School of Brown University, Providence, Rhode Island, USA

${ }^{3}$ Department of Emergency Medicine, University of Colorado School of Medicine, Denver, Colorado, USA

${ }^{4}$ Department of Emergency Medicine, Emory Healthcare Network, Atlanta, Georgia, USA

${ }^{5}$ Department of Pediatrics, Medical College of Wisconsin, Milwaukee, Wisconsin, USA

${ }^{6}$ Departments of Pediatrics and Global Health, University of Washington, Seattle, Washington, USA

${ }^{7}$ Department of Emergency Medicine, Brigham and Women's Hospital, Boston, Massachusetts, and Harvard Humanitarian Initiative, Cambridge, Massachusetts, USA

${ }^{8}$ Department of Emergency Medicine, University of Florida, Gainesville, Florida, USA

${ }^{9}$ Usher Institute of Population Health Sciences and Informatics, University of Edinburgh, Edinburgh, UK

*Joint senior authors.
}

\section{Correspondence to:}

Walter Tyler Winders, MD MPH

4009 Campbell St

Kansas City, MO 64110

USA

tylerwinders@gmail.com
Background In resource-constrained settings, mobile health (mHealth) has varied applications. While there is strong evidence for its use in chronic disease management, the applications of mHealth for management of acute illness in low- and middle-income countries (LMICs) are not as well described. This review systematically explores current available evidence on the effectiveness of mHealth interventions at improving health outcomes in emergency care settings in LMICs.

Methods A systematic search of the literature was performed in accordance with PRISMA guidelines, utilizing seven electronic databases and manual searches to identify peer-reviewed literature containing each of three search elements: mHealth, emergency care (EC), and LMICs. Articles quality was assessed using the Grading of Recommendations Assessment, Development and Evaluation (GRADE) criteria.

Results After removing duplicates, 6498 studies met initial search criteria; 108 were eligible for full text review and 46 met criteria for inclusion. Thirty-six pertained to routine emergency care, and 10 involved complex humanitarian emergencies. Based on the GRADE criteria, 15 studies were rated as "Very Low" quality, 24 as "Low" quality, 6 as "Moderate" quality, and 1 as "High" quality. Eight studied data collection, 9 studied decision support, 15 studied direct patient care, and 14 studied health training. All 46 studies reported positive impacts of mHealth on EC in LMICs.

Conclusions Mobile health interventions can be effective in improving provider-focused and patient-centered outcomes in both routine and complex EC settings. Future investigations focusing on patient-centered outcomes are needed to further validate these findings.

Mobile phones have evolved from simple communication devices to smartphone platforms with advanced data storage, internet access, and photographic capabilities. These emerging technologies have been widely adopted in health care and fall under the inclusive term mobile health (mHealth), defined by the World Health Organization (WHO) as "medical and public health practice supported by mobile devices, such as mobile phones, patient monitoring devices, personal digital assistants, and other wireless devices." [1]. Mobile health advances have enabled cutting edge health care in traditionally resource-constrained environments [2]. 
Mobile health applications improve upon the disproportionately poor health outcomes that low- and- middle-income countries (LMICs) are known to endure [2]. The evidence base for mHealth in LMICs is robust but largely focused on chronic care. There are numerous systematic reviews and meta-analyses detailing the effectiveness of mHealth on the care for various chronic diseases in LMICs, revealing improved medication adherence, symptom control, and lowered risk of death and hospitalization [3-5]. However, mortality associated with routine emergency care (EC) in LMICs is several-fold higher than in high-income countries (HICs) [6,7], and complex humanitarian emergencies (CHEs) are known to exhibit extremely high mortality rates, particularly when they occur in an LMIC [8]. These well documented inequities lend themselves to innovative interventions, such as mHealth. There is a growing body of literature on the use of mHealth for addressing acute care needs in LMICs that has yet to be synthesized. Initial studies on the utility of mHealth in EC in LMICs are promising. The objective of this systematic review is to provide the first formal synthesis of the effects of mHealth as it relates to emergency care in LMICs.

\section{METHODS}

\section{Search strategies}

This systematic review was developed and conducted in collaboration with the Global Emergency Medicine Literature Review (GEMLR) group and the Usher Institute of Population Health Sciences and Informatics (University of Edinburgh). The Usher Research Ethics Group approved systematic review protocol, which was then registered with PROSPERO (registration number CRD42019151080) on December 20, 2019. The published study protocol is available in Appendix S1 of the Online Supplementary Document and searchable on PROSPERO. A rigorous search strategy was designed in collaboration with a health sciences medical librarian with the goal of identifying all randomized controlled trials and observational studies that described the effectiveness of mHealth interventions on EC in LMICs. No patients were asked for input in the creation of this article.

Five unique electronic databases were systematically searched: Pubmed, OVID: Global Health (CABI), Embase, Web of Science, and Global Index Medicus. These databases were selected with the goal of including all published literature worldwide. Eligible studies included all randomized controlled trials (RCTs) and observational studies with controls that included each of three major search themes: mobile health, emergency care, and LMIC (see Appendix S2 in the Online Supplementary Document). The initial search strategy was developed within Pubmed and adapted for the remaining four databases. Free text terms and standardized MeSH headings/subheadings in the context of Boolean operators and appropriate search term truncation were utilized to optimize sensitivity for relevant literature while minimizing excess search results. The search strategy was optimized via multiple trial searches, verifying that all previously identified relevant studies were included. The reference lists of prior similar reviews and studies likely to meet inclusion criteria were searched manually to both verify search sensitivity and identify other potentially relevant studies that were not identified by the electronic search. A manual grey literature search was also performed via advanced Google searches targeting organizations (eg, World Health Organization and International Committee of the Red Cross) known to publish global EC literature. See Appendix S2 in the Online Supplementary Document for the specific search strategies utilized for each database.

\section{Data processing}

After removal of duplicate articles, two independent reviewers screened each title and abstract. Covidence, an online systematic review platform, was utilized to facilitate study screening [9]. The primary author (WTW) screened all titles and abstracts, and second reviewers (CB, SG, PR, MS) each screened one quarter. Discrepancies were resolved by a third reviewer from the GEMLR group. The same procedure was followed for full-text screening. Articles were excluded if they were not in English or Spanish, clearly irrelevant to the topic (such those with a focus on chronic disease management and/or care in high-income countries), not emergency care, considered social media (internet based applications, such as WhatsApp or Twitter, that allow exchange of user created content [10]), not mHealth, evaluating non-health care related outcomes (such as economic/ monetary outcomes, supply chain efficiency, management or process outcomes), not undertaken in a LMIC, or abstract-only publications.

Data extracted from final manuscripts included: author, publication date, location, study type, mHealth intervention type, setting (routine EC or CHE), methods, primary outcomes, secondary outcomes, and limitations. Study quality was assessed using the Grading of Recommendations Assessment, Development, and Evaluation (GRADE) criteria [11]. Criteria proposed by the Preferred Reporting Items for Systematic Reviews and Meta-analyses (PRISMA) statement were adhered to in reporting [12]. 


\section{Data analysis}

The study aims resulted in significant heterogeneity in study type, methodology, intervention, and outcomes. Even when studies were stratified into four categories of mHealth interventions (decision support, data collection, direct patient care, and health education), significant heterogeneity remained. The requisite criteria for formal meta-analyses and funnel plots were not met, and therefore, a qualitative analysis and narrative synthesis was undertaken. Thematic analysis was undertaken focusing on the effectiveness of the health outcomes in each of the four different categories of mHealth applications in EC in LMICs.

\section{RESULTS}

A total of 8947 articles were identified for screening via database searches, and 12 were included based on the grey literature search (Figure 1). After removal of duplicates, 6495 unique articles were included in title and abstract screening. A total of 108 articles met criteria for full text screening. 62 articles were excluded during full text screening, leaving 46 articles that met criteria for inclusion.

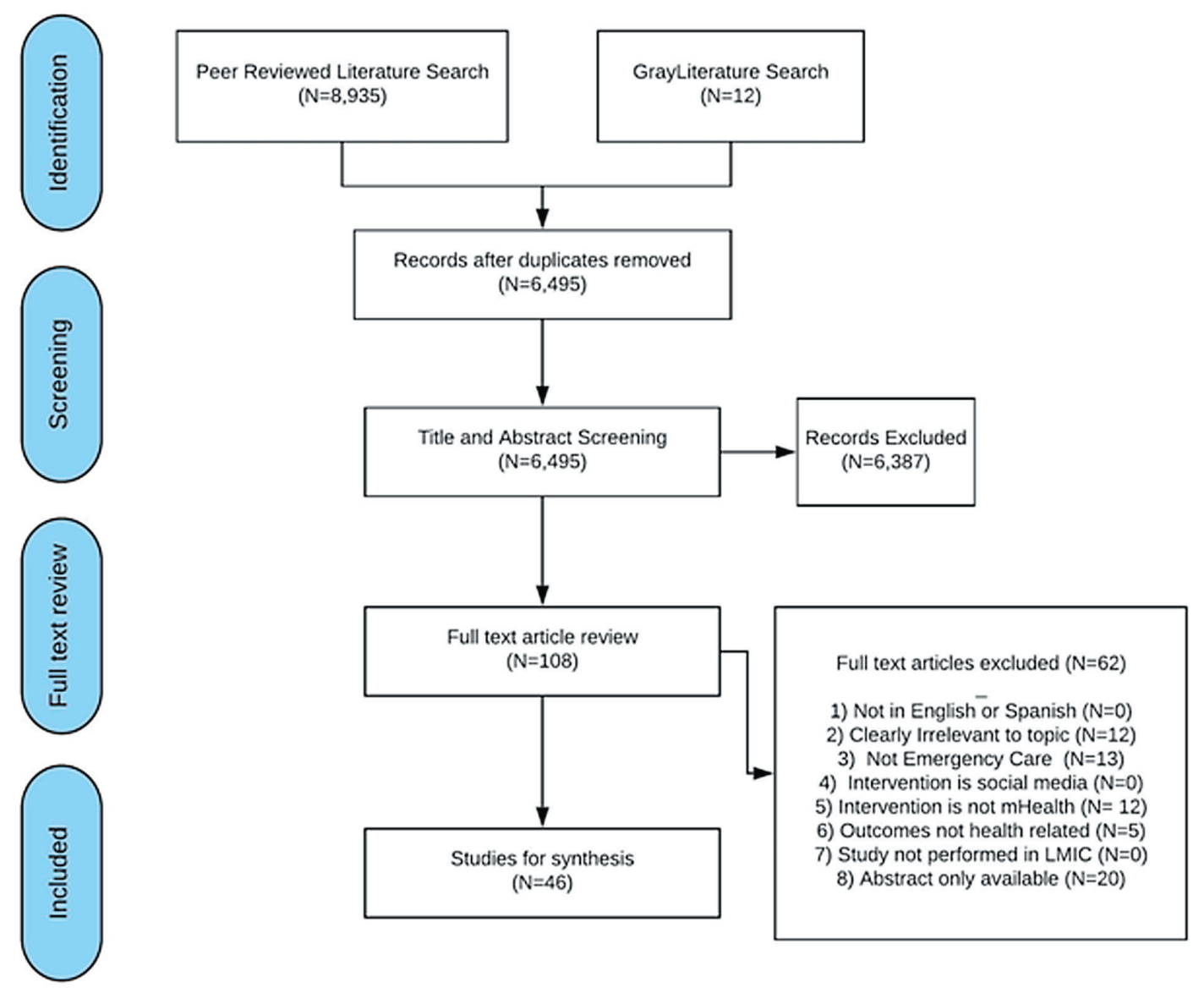

Figure 1. PRISMA flow diagram.

Of the 46 included studies, 45 were published in peer-reviewed journals, and one was a grey literature publication. Thirty-six encompassed routine EC, and 10 involved CHEs. Geographically, publications originating from all six WHO Member State Regions were included [13]. Based on the World Bank development indices, this systematic review encompassed 16 studies from low-income countries, 12 studies from lower-middle income countries, and 17 studies from upper-middle income countries. A single study reported data from three distinct countries, World Bank development classifications, and regions [14]. Table 1 provides general study characteristics.

Methodologically, 7 studies were randomized controlled trials (RCTs), 21 were observational cohorts with control groups, and 18 were observational pre/post studies. Of the four prespecified categories of mHealth interventions delineated in this systematic review, 8 studied data collection interventions [15-22], 9 studied de- 
Table 1. Study characteristics

\begin{tabular}{lc} 
WHO REGIONAL OFFICE* & $\mathbf{N}(\%)$ \\
AFRO & $19(42.2 \%)$ \\
\hline AMRO & $7(15.5 \%)$ \\
\hline SEARO & $10(22.2 \%)$ \\
\hline EURO & $2(4.4 \%)$ \\
\hline EMRO & $4(8.8 \%)$ \\
\hline WPRO & $3(6.6 \%)$ \\
\hline Lorld Bank Development Index & \\
\hline Lower-middle income & $16(35.5 \%)$ \\
\hline Upper-middle income & $12(26.6 \%)$ \\
\hline Setting & $17(37.7 \%)$ \\
\hline Complex humanitarian crisis & $10(21.8 \%)$ \\
\hline Routine emergency care & $36(78.2 \%)$ \\
\hline
\end{tabular}

\begin{tabular}{lc}
$\begin{array}{lc}\text { WHO REGIONAL OFFICE* } \\
\text { Methodology }\end{array}$ & N (\%) \\
\hline RCT & $7(15.2 \%)$ \\
\hline Observational cohort & $21(45.7 \%)$ \\
\hline Observational pre/post & $18(39.1 \%)$ \\
\hline mHealth intervention & $8(17.4 \%)$ \\
\hline Data collection & $9(19.6 \%)$ \\
\hline Decision support & $15(32.6 \%)$ \\
\hline Direct patient care & $14(30.4 \%)$ \\
\hline Health trainings & $1(2.2 \%)$ \\
\hline GRADE quality & $6(13.0 \%)$ \\
\hline High & $24(52.2 \%)$ \\
\hline Moderate & $15(32.6 \%)$ \\
\hline Low
\end{tabular}

WHO - World Health Organization, AFRO - African Regional office, AMRO - American regional office, SEARO - South-East Asian regional office, EURO - European regional office, EMRO - Easter Mediterranean regional office, WPRO - Western Pacific regional office, RCT - randomized controlled trial

*Finette et al. reported data from Burkina Faso (AFRO/low income), Ecuador (PAHO/upper-middle income), and Bangladesh (SEARO/ lower-middle income) [14]

cision support interventions [14,23-30], 15 studied direct patient care interventions [31-45], and 14 studied health training interventions [46-59]. Table 2 provides detailed study characteristics. The four prespecified subtypes of mHealth interventions were utilized to structure the thematic analysis.

\section{Data collection}

The 8 studies in this category include 4 that were undertaken during humanitarian crises: two during the 2010 Haiti earthquake and two during the 2014-2016 West African Ebola epidemic. The Haitian studies report on the effectiveness of mobile phone sim card data to both predict population movements and infectious outbreak pressure in humanitarian crisis settings [15,16]. Mobile-phone based models outperformed traditional large data sets, and the calculated infectious pressure at outbreak onset was significantly correlated with reported cholera cases during the first ten days of the epidemic. Population level epidemiological monitoring was improved in all four studies evaluating mHealth data collection tools in the setting of disease outbreaks, such as EVD or malaria. When compared to the standard paper method, there was improved timeliness of submission and data completeness $[17,18,21,22]$. Two observational studies found short messaging service (SMS) an effective means to obtain individual level symptom and health seeking data $[19,20]$. Specifically, individual-level SMS messaging was used to monitor EVD symptoms and found to have a strong positive linear correlation between cell phone syndromic surveillance and reported EVD suspect cases and deaths. Mobile health data collection interventions appear to be effective on population and individual levels as compared to traditional paper or door-to-door data collection methods.

\section{Decision support}

These 10 studies include a single RCT and nine observational studies that evaluated smartphone-based triage tools, evidence-based smartphone algorithms, and provided diagnostic support from specialists. To assess the feasibility of decision support instruments, a mixed-methods study utilized self-assessments to evaluate a neonatal care decision support application. Healthcare workers (HCWs) reported improved clinical confidence and perceived improvements in the quality of newborn care [26]. Further, a before-and-after study with a similar neonatal resuscitation support application was employed in a Uganda labor and delivery unit. This study employed trained third party observers to evaluate neonatal resuscitation and found the mean adherence to the standards of neonatal resuscitation to increase from $46 \%$ pre-intervention to $94 \%$ post-intervention [27]. Mobile health applications for patient triage outperformed standard paper assessments in acutely ill children and adults. These studies found improved accuracy in the triage of general ED patients in Thailand, acutely dehydrated patients with diarrhea in Bangladesh, and in a multinational cohort of children $<5$ years of age as compared to the gold standard paper triage assessments $[14,29,30]$. Finally, a single study found somewhat limited utility of remote visual diagnostics in pediatric burn patients. Remote specialists had highly accurate total body surface area (TBSA) estimates but poor depth assessments [25]. All nine studies concluded that mobile health decision support interventions were an effective means to improve EC in LMICs. 


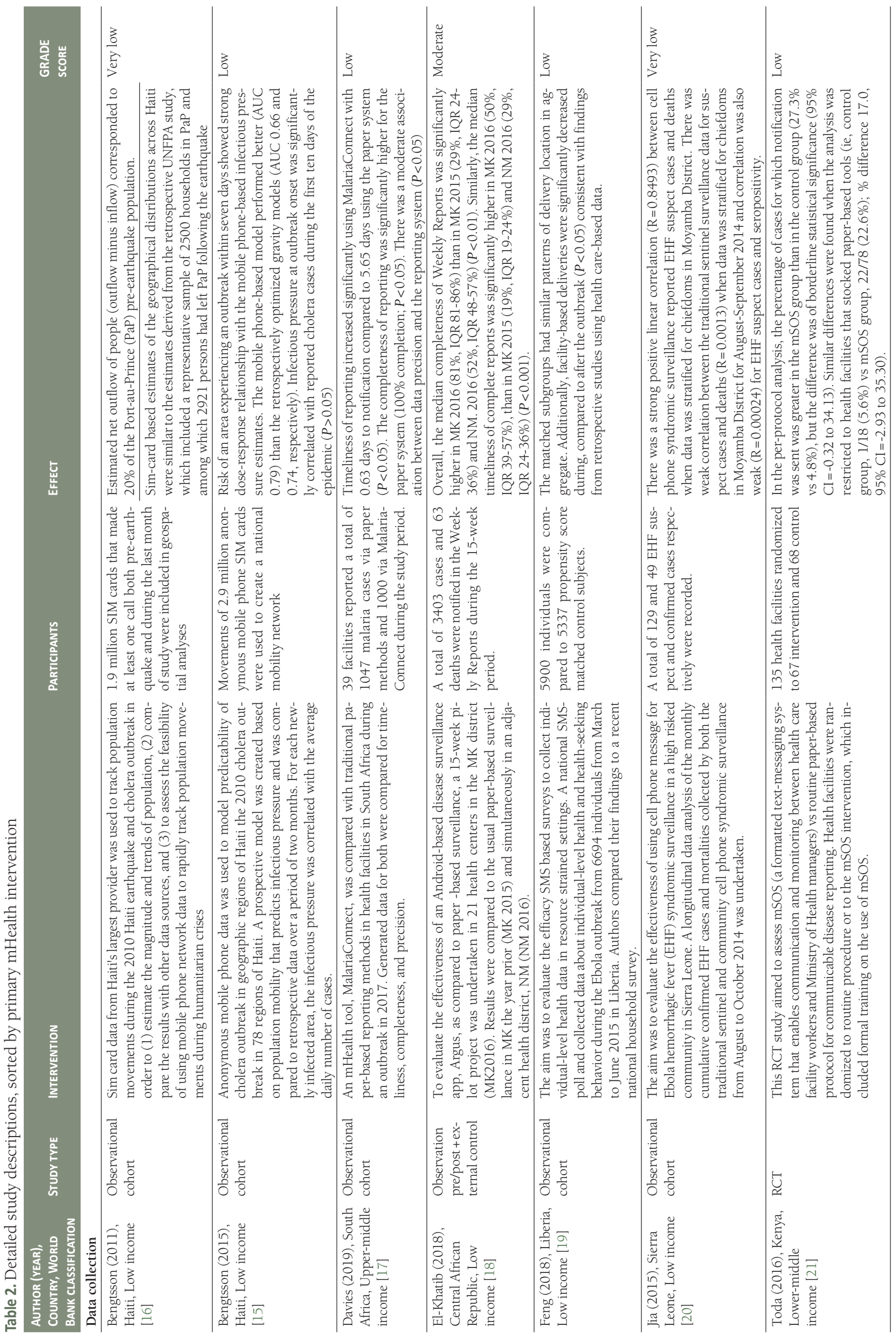




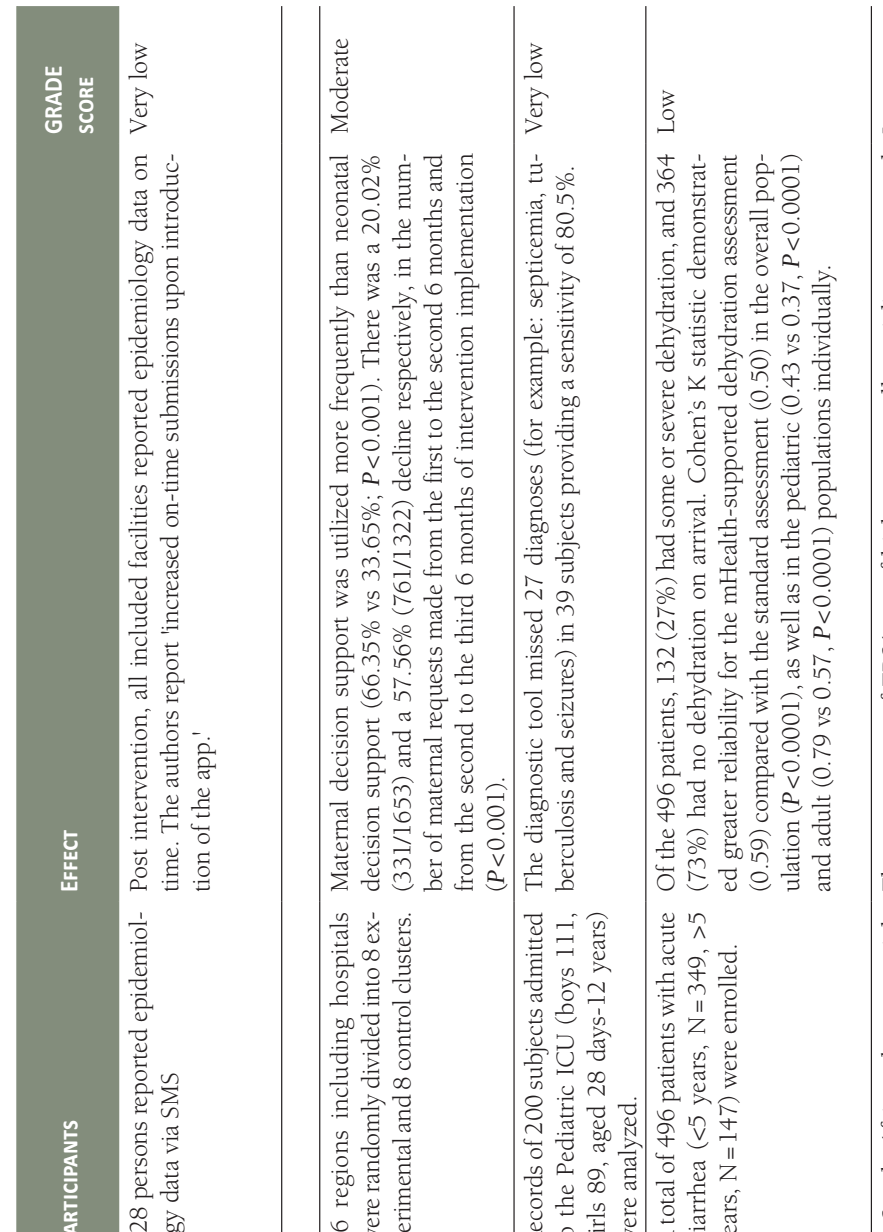

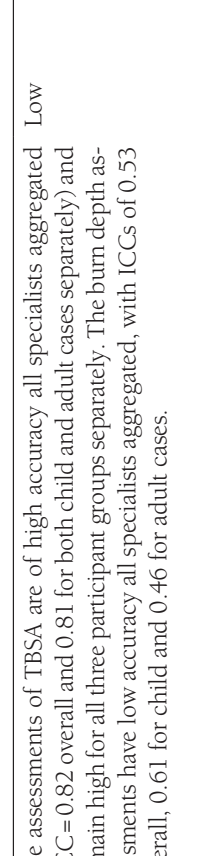

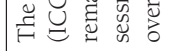

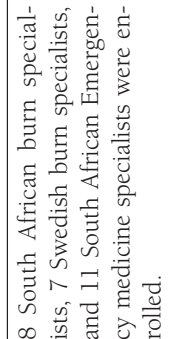

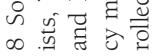

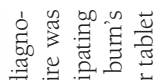

ت气. :

है을

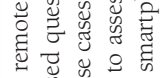

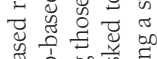

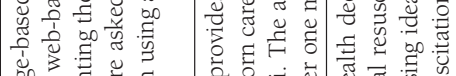

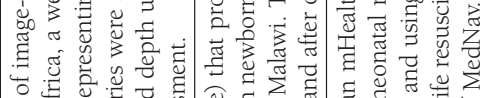

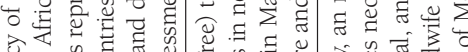

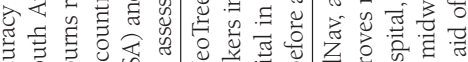

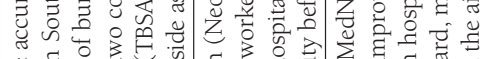

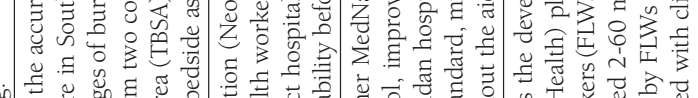

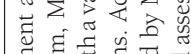

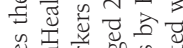

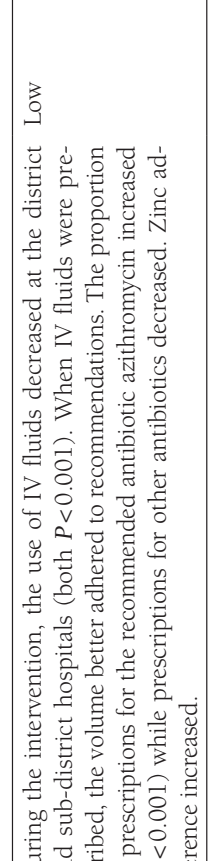

త⿹丁口⿹丁口

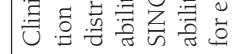

ก.

芯

范

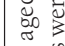

플

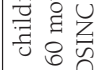

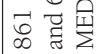

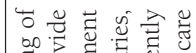

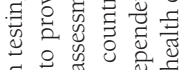

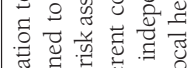

疍

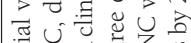

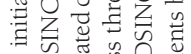

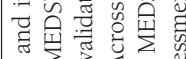

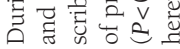

这 운

范

ํㅗㄹ

竞

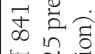

पै तै

焉

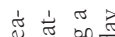

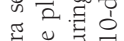

일

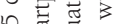

ते है है.

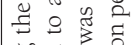

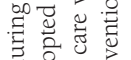

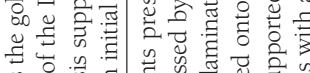

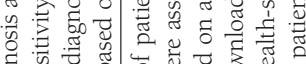

胥

苛苛

告言䓪

诘芯

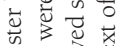

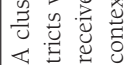

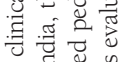

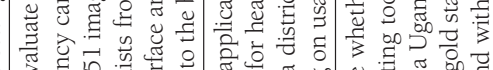

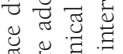

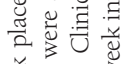

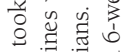

둔

न

정 을

它 돌

둥

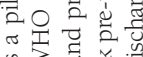

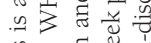

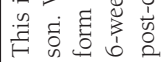
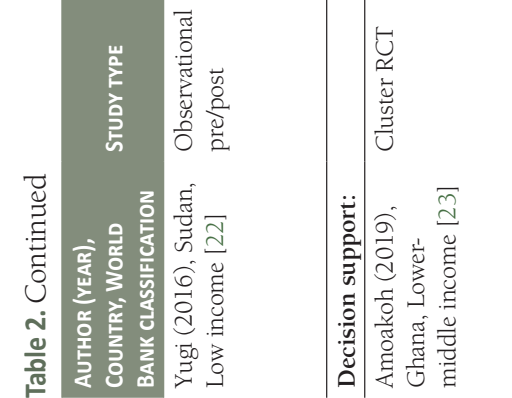

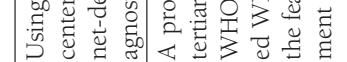

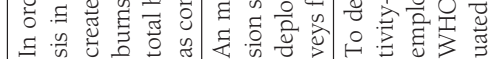
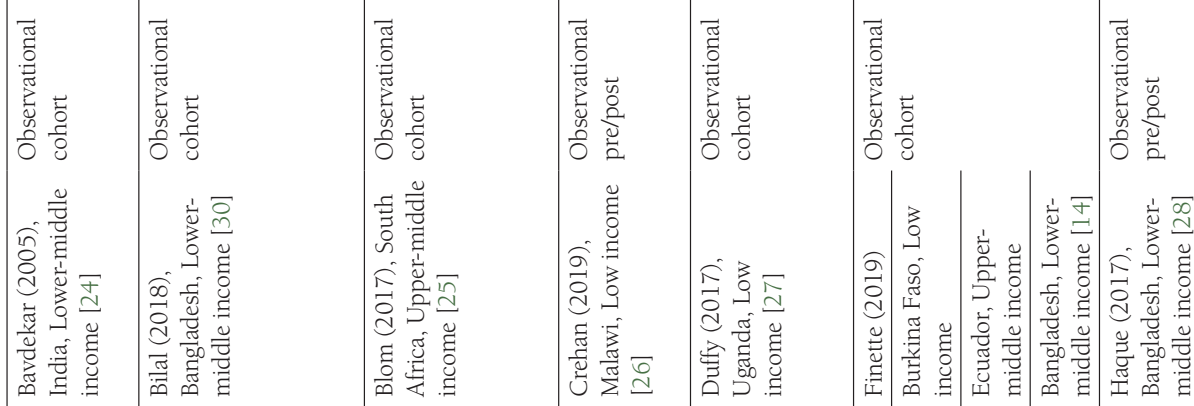


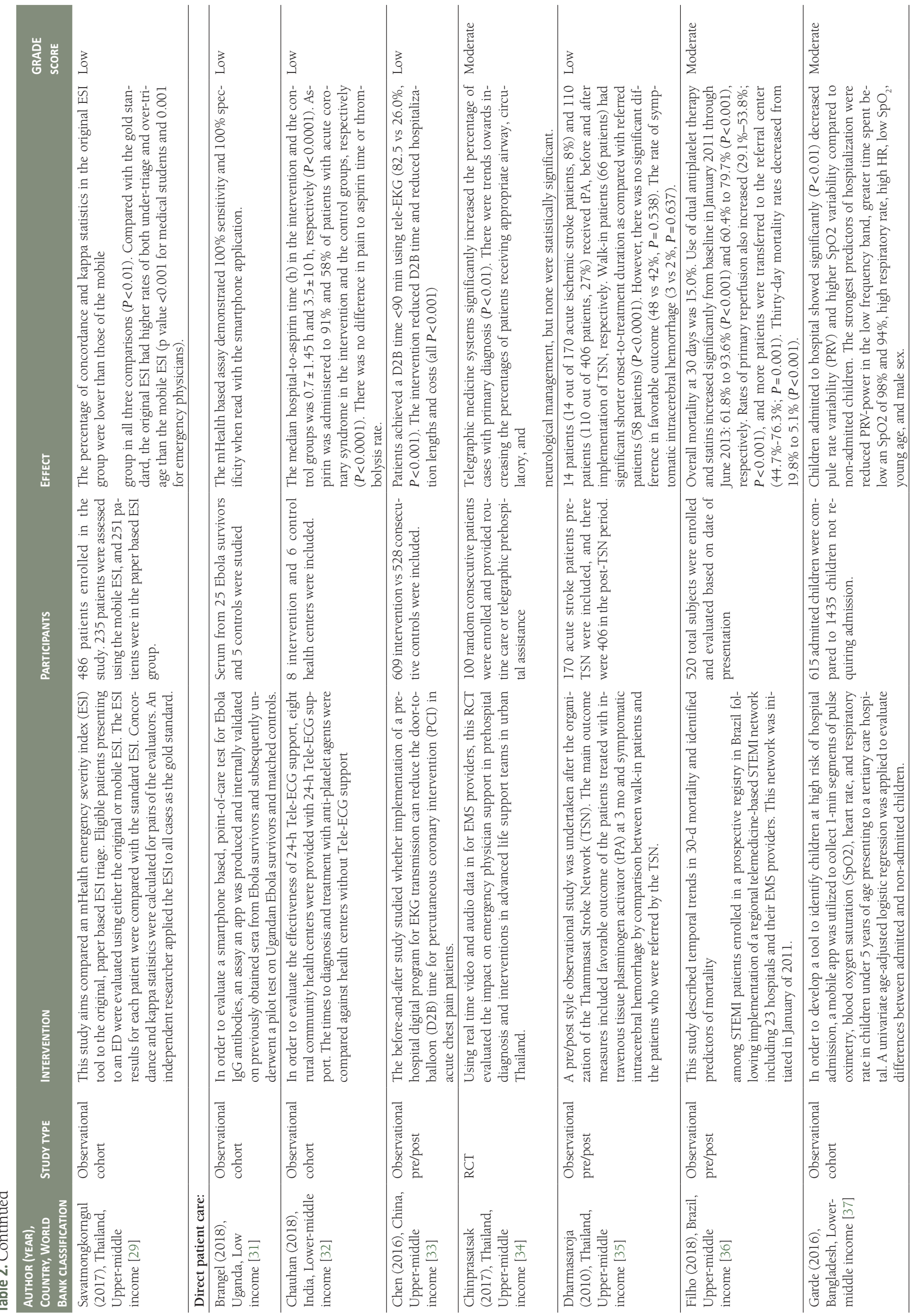




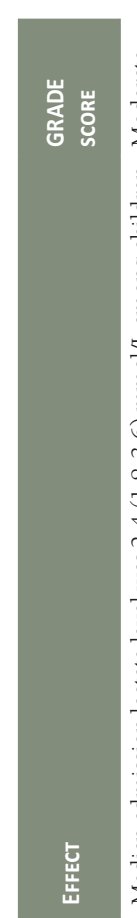

营

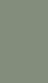

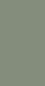

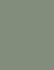

$\vec{\square}$

政

衰

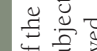

范言

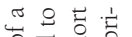

苞艺

䆑票吉

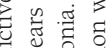

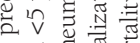

气

on

突 50.0

क ज च

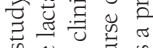

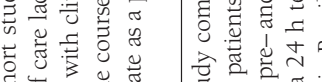

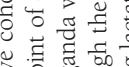

芴

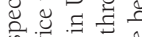

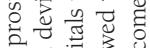

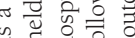

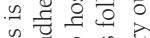

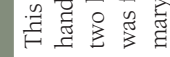

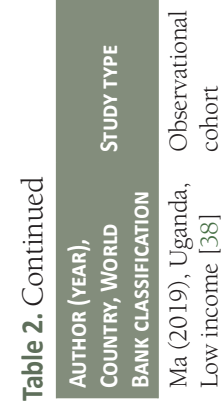

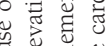

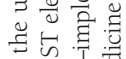

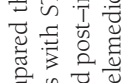

$\left|\frac{3}{3}\right| \frac{3}{3}$

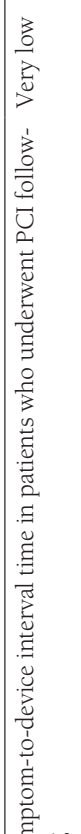

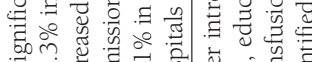

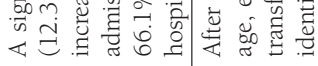

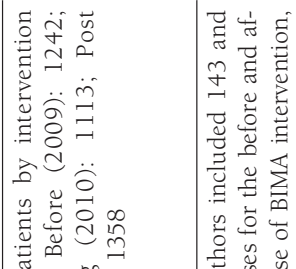

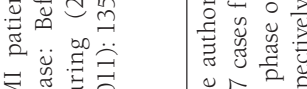

专鱼言亭

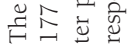

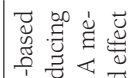

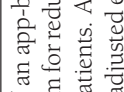

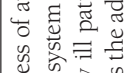

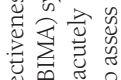

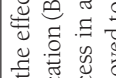

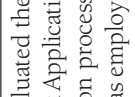

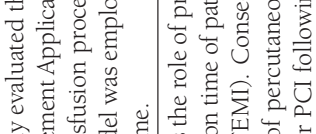

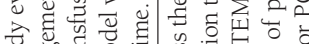

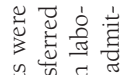

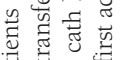

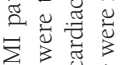

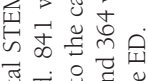

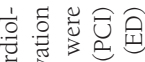

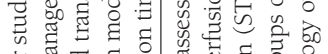

$\frac{3}{3}$

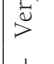

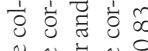

童

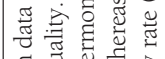

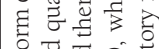

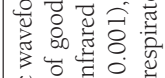

苞艺 $v$

勘

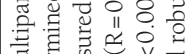

范萬

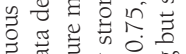

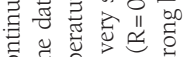

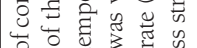

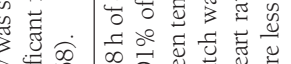

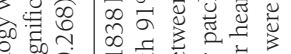

잉

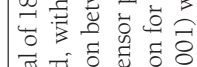

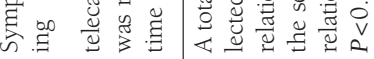

気 突

它 $\sum_{\sum}^{n}$

总交

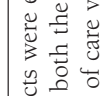

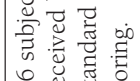

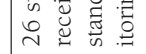

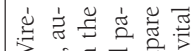

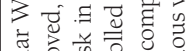

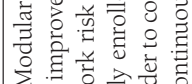

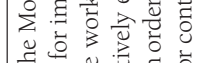

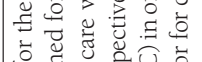

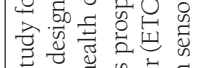

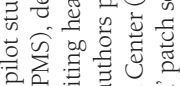

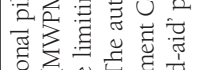

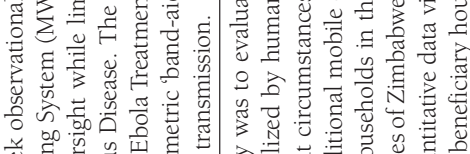

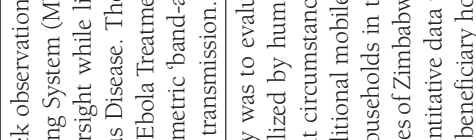

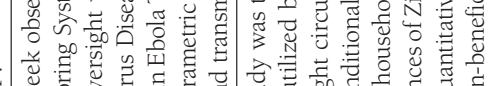

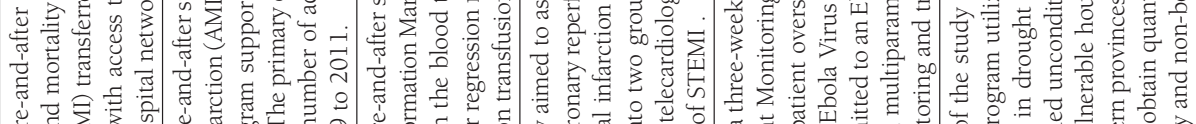

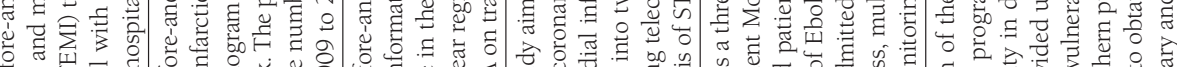

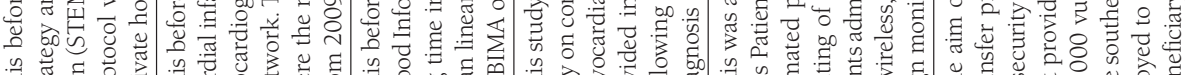

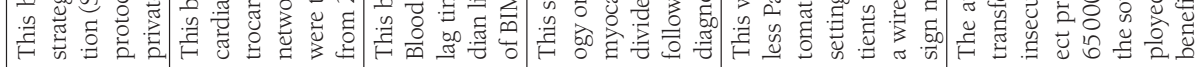

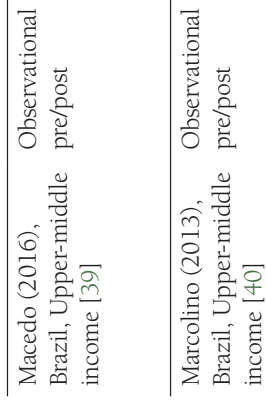

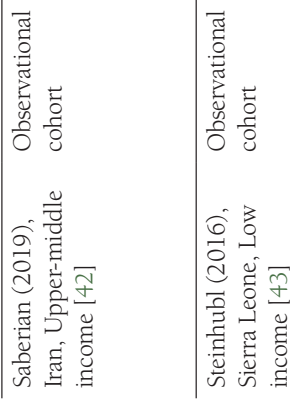

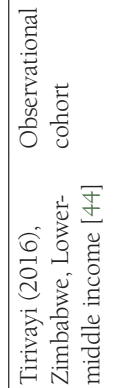

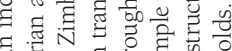

高

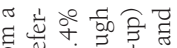

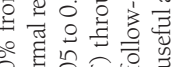

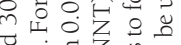

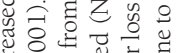

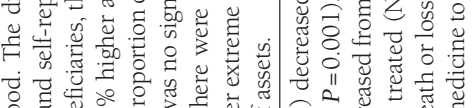

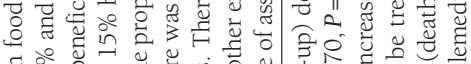

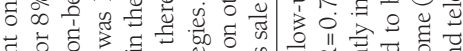
泀.气

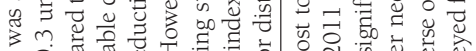

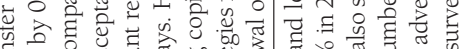

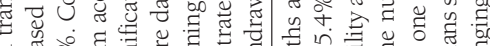

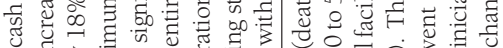

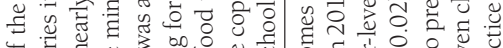
․ㅠ.

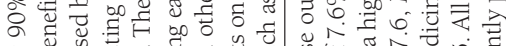

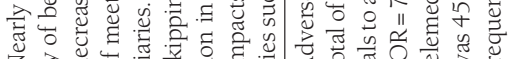
तें 렬

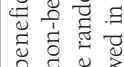

존

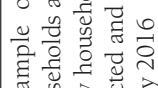

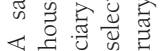

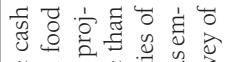

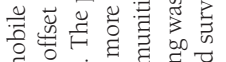

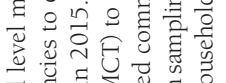

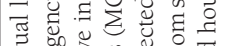

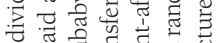

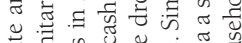

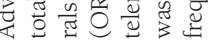

$\exists \overparen{\natural}$

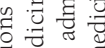

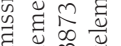

ت

范势

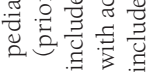

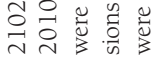

实常苞导 के

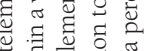

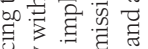

昰焉要

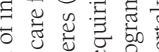

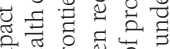

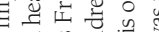

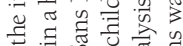
w

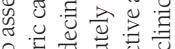

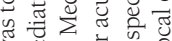

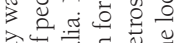

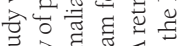
जी

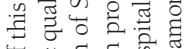

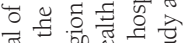
๙ั.

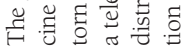

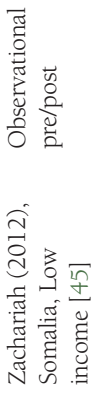




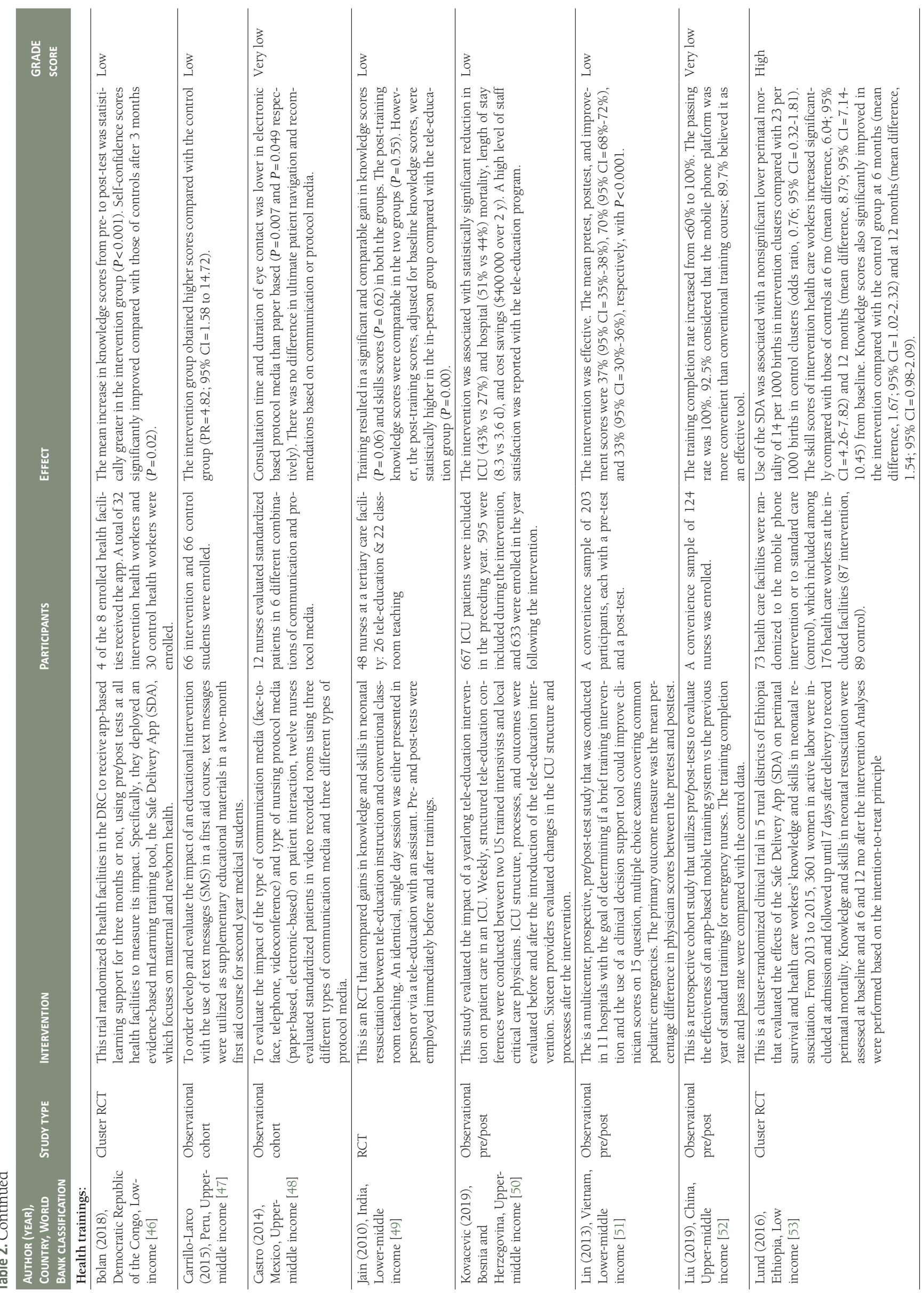




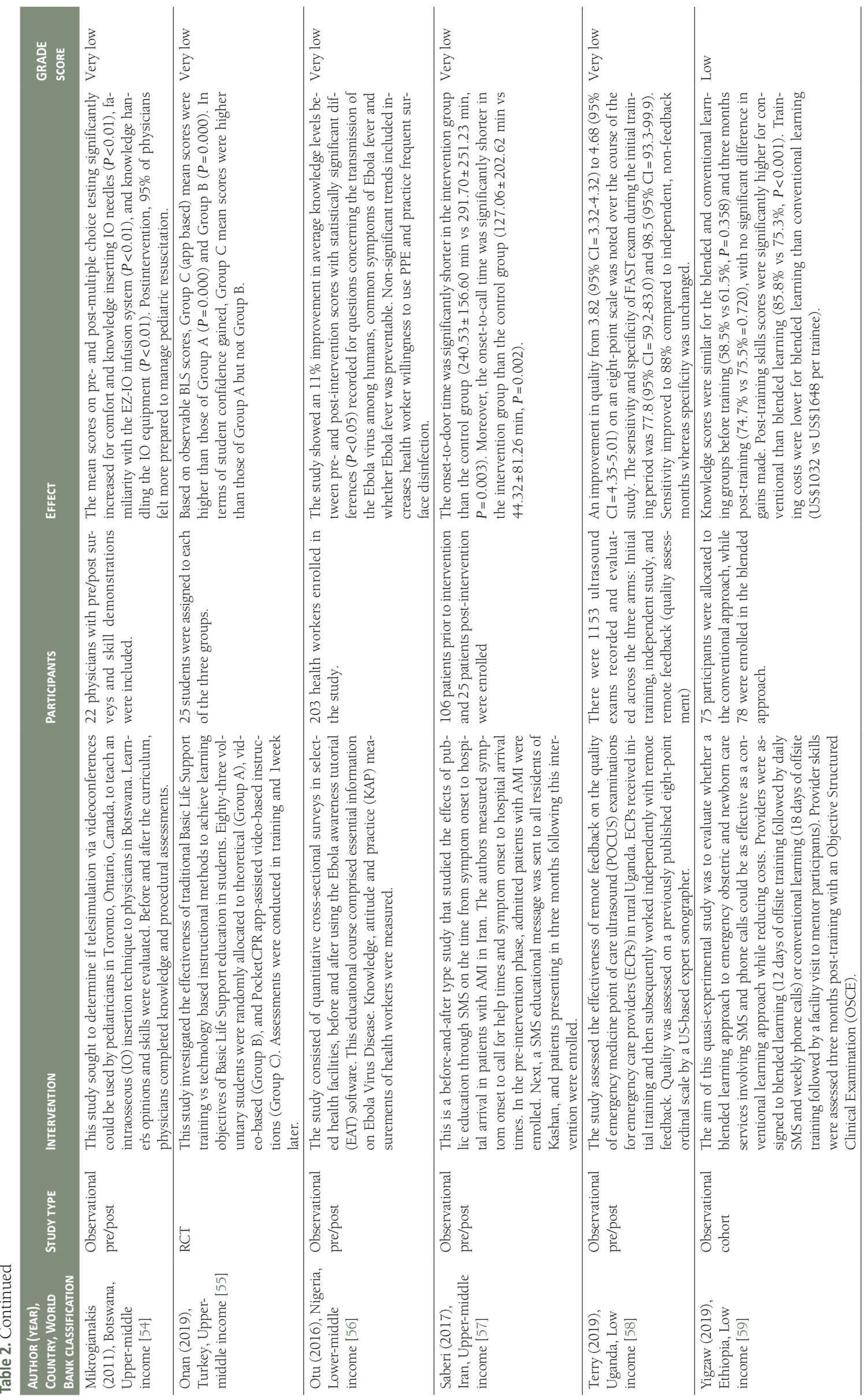




\section{Direct patient care}

The 15 studies in this category include a single RCT and 14 observational trials. Six observational studies evaluated novel technologies such as point-of-care (POC) testing [31,38], patient monitoring devices [37,43], or app-based health resource management $[41,44]$. A pilot study evaluated the ability of smartphone cameras coupled with an mHealth app to perform a POC assay for Ebola IgG antibodies. It found 100\% sensitivity and $100 \%$ specificity on a cohort of 25 known positive subjects and 5 controls [31]. Utilizing a novel, stand-alone mobile instrument, a prospective cohort of 150 children $<5$ years of age requiring admission in Uganda found POC lactate testing to be a better prognostic marker of mortality, than any single clinical sign or composite clinical risk score [38]. A mHealth app studied in Bangladesh collected minute-long segments of pulse oximetry, blood oxygen saturation $\left(\mathrm{SpO}_{2}\right)$, heart rate, and respiratory rate in children under 5 years of age presenting to a tertiary care hospital. This application found pulse rate variability and hypoxia to be the two strongest predictors of admission [37]. Another prospective cohort compared a novel patient monitor device in an Ebola Treatment Center to standard nurse taken vital signs and displayed strong correlations for temperature, heart rate, and respiratory rate [43]. Finally, an mHealth application produced a 24-minute reduction in the time was observed between the identified need for blood and transfusion, as compared to standard paper systems [41].

The remaining nine studies encompassed telehealth interventions that took place in all phases of care from patient homes to the prehospital arena to health facilities, including a single telehealth study in a CHE. Telecardiology support for HCWs, in particular, was a recurrent theme encountered in this sector of mHealth. Six observational studies evaluating tele-cardiology support in acute coronary syndrome for prehospital providers or other HCWs such as emergency or primary care providers demonstrated significantly improved rates of administration of aspirin (91\% vs 58\%), improved rates of primary reperfusion, faster door-to-balloon (D2B) time for STEMI (D2B time <90 minutes: 82.5 vs $26.0 \%$ ), a reduction of in-hospital mortality rates (12.3\% vs $7.1 \%)$, and a reduction in thirty-day mortality rates (19.8 vs 5.1\%) [32,33,36,39,40,42]. The lone RCT in this subgroup randomized ambulances staffed with EMS providers to real-time telehealth support vs standard care for the rate of correct primary diagnosis in the prehospital arena. They demonstrated significant improvement in arriving at the correct prehospital diagnosis and demonstrated nonsignificant trends towards improved the percentage of patients receiving appropriate prehospital management [34].

\section{Health training}

A total of 14 studies described the use mHealth technologies for an educational purpose. These include four RCTs and 10 observational studies. The most common primary outcome in this subgroup was HCW knowledge or skills improvement. This group of studies utilized pre- and post-tests in cohorts of HCWs to demonstrate that mHealth interventions are an effective means to improve HCW knowledge and skills in various emergency capacities such as neonatal resuscitation, point of care ultrasonography (POCUS), and EVD knowledge $[46,47,49-56,58,59]$. An RCT evaluated the impacts of an educational mHealth app on perinatal survival in addition to HCW knowledge and skills in 73 health facilities in Ethiopia. The authors found that use of the application was associated with a nonsignificant lower perinatal mortality of 14 per 1000 births in intervention clusters compared with 23 per 1000 births in control clusters but did find significant improvements in HCW skills and knowledge [53]. A similar mHealth intervention was applied in a public health context. This observational study on SMS-based education in lay persons in Iran found that educational messages on acute coronary syndrome symptoms decreases both the symptom onset to ED arrival and call for help times [54].

\section{Complex humanitarian emergency}

Of all 46 included studies, 10 publications evaluated the role of mHealth in CHEs. Half of these were performed during the 2014 Ebola outbreak, two were performed during the 2010 Haitian earthquake, and three took place in the setting of civil unrest and profound food insecurity. Specific to the 2014 Ebola outbreak, an observational study demonstrated that an mHealth based educational program was an effective way to improve health worker knowledge on EVD [56]. Further observational publications demonstrated that SMS messaging was effective on the individual level for monitoring health care behaviors and symptom development, respectively $[19,20,48]$. Two publications demonstrated the utility of novel patient care devices, piloting the use of a wearable remote patient vital sign monitor and a point-of-care EVD IgG test respectively $[31,43]$. Post-Haitian earthquake, two large observational data sets demonstrated the utility of anonymous cell phone data in both tracking population movements and predicting regional infectious disease outbreaks based on these movements $[15,16]$. To evaluate the impact of mHealth on epidemic surveillance during a CHE, a before-and-after study demonstrated that mHealth tools result in significantly improved completeness and timeliness as compared to traditional paper-based methods [18]. A before-and-after study evaluated the impact of an Médecins Sans 
Frontiérs (MSF) telehealth intervention on patient care in a war-torn region of Somalia. Over two years, this study enrolled nearly 6000 acutely ill children requiring hospital admission and demonstrated that telehealth support from Kenyan pediatricians significantly decreased the risk of death or loss to follow up [45]. Finally, the impact of United Nations supported mobile cash transfer program on diet and hunger in drought-affected communities in Zimbabwe was evaluated prospectively via random household surveys. 90\% of the cash transfers were spent on food, which improved dietary diversity, decreased self-reported, and significantly improved the probability of meeting the minimum acceptable dietary standards [44].

\section{Patient-centered outcomes}

Twelve (26\%) of the 46 total studies were patient-centered in nature. Six studies reported the impact of mHealth interventions on mortality. Four observational cohorts found their respective interventions to decrease mortality rates $[36,40,45,50]$. Two before-and-after observational trials evaluating regional telecardiology programs in Brazil found these system wide interventions significantly increased rates of primary reperfusion in STEMI (29.1 vs $53.8 \%$ ) and ICU admission (32.4 vs $66.1 \%$ ) while decreasing both thirty-day mortality rates (19.8 seconds, $5.1 \%$ ) and in-hospital mortality rates (12.3 vs $7.1 \%$ ) [36,40]. A yearlong ICU-based study evaluated weekly tele-education conferences focused on ICU structure, processes, and outcomes in Bosnia and Herzegovina. This before-and-after study enrolled approximately 600 patients yearly over 3 years and demonstrated statistically significant reductions in ICU mortality ( $43 \%$ vs $27 \%$ ), in-hospital mortality ( $51 \%$ vs $44 \%$ ), and length of stay (8.3 vs 3.6 days), over the study period [50]. The MSF supported before-and-after study described above demonstrated that telehealth support decreases mortality in pediatric patients requiring hospital admission in a CHE [45]. Two studies reported non-significant trends towards decreased mortality [39,53]. The lone RCT with patient centered outcomes evaluated the impacts of an educational application on perinatal mortality and did not demonstrate a statistically significant mortality benefit. Extrapolating that door-to-balloon (D2B) time and appropriate pharmacologic management of acute coronary syndrome (ACS) is associated with a reduced risk of death [60], three additional studies demonstrated favorable patient outcomes by proxy [32,33,57]. A Thailand based tele-stroke focused study demonstrated increased adherence to guideline-based use of tPA via a tele-stroke network but found no difference in favorable neurologic outcomes at 3 months [61].

\section{DISCUSSION}

The studies included in this review demonstrated a positive impact of mHealth interventions on the quality of EC in LMICs. The measured outcomes are diverse and largely showed improvement of provider-focused and patient-centered outcomes regardless of setting or subset of mHealth intervention (data collection, decision support, direct patient care, or health training). These findings are in line with previous systematic reviews examining the impacts of mHealth in LMICs in disciplines outside of EC, such as chronic disease management [62-64].

The mHealth studies on data collection included in this systematic review demonstrated improved epidemic monitoring data quality and timeliness in both routine EC and complex humanitarian emergencies, such as the 2014-16 EVD outbreak, compared to traditional paper standard. On the individual level, mHealth applications may effectively monitor both symptomatology and health resource utilization. On the population level, mHealth interventions may effectively predict population movement and infectious disease outbreak risks, enabling public health officials to anticipate and intervene in potentially problematic regions.

Decision support studies report satisfactory clinical utility and reliability of a variety of decision support algorithms targeted for HCWs. These studies aimed at improving health care quality by supporting HCWs with evidence-based medicine algorithms; however, they focused primarily on usability or HCW satisfaction with their respective mHealth interventions. Two studies did report improved HCW adherence to established international treatment guidelines, which may improve clinical care by proxy $[27,28]$.

Direct patient care was the most robust subset of mHealth studies included in this systematic review. Based on average GRADE scores, this subset has a higher percentage of quality publications. The majority were telehealth interventions, which demonstrated a high proportion of positive patient-centered outcomes. The tele-cardiology studies generally demonstrated improved patient outcomes and clinical care consistent with prior publications from HICs [65]. The lone tele-stroke study improved rates of tPA administration [66]. The remaining studies in this subset consistently demonstrated the utility of novel technologies in EC in resource limited settings. From novel patient monitors in EVD units [67] to advanced point-of-care diagnostics [37,38], these studies showcased mHealth as a means to bring a high-quality and equitable level of care to remote, resource-constrained environments. 
Finally, each of the 14 included educational studies conclude that mHealth is an effective means of $\mathrm{EC}$ health training in LMICs. This cohort of studies demonstrated that educational content does not always require traditional face-to-face teaching to improve provider knowledge and quality of care. In fact, mHealth supplementation may be superior to traditional teaching [47] and improves course completion rates [52]. Further, it may even improve patient outcomes by helping to ensure the delivery of consistent and high-quality care while increasing population access to health services [50]. Importantly, the majority of these studies did include formal curricula. Taken as a whole, these studies suggest that mHealth is an effective means to support educational needs in resource limited settings.

Notably, patient centered outcomes were generally lacking. Only one quarter of the studies measured patient-centered outcomes, which highlights the need for an increased focus on the important downstream impacts of mHealth interventions in the future.

\section{Limitations}

Publication bias is likely the largest limitation of the literature found in this systematic review. The lack of negative studies encountered, particularly when evaluating novel technologies in resource-limited settings, may imply that many negative studies simply are not being published. Additionally, several studies include mHealth interventions, such as telecardiology, as part of a larger systemic intervention, making it impossible to distinguish to isolate effect of the mHealth component on endpoints such as mortality. These studies potentially introduce unmeasured confounding covariates that may skew the actual relationship between mHealth and the primary outcomes.

Methodologically, this systematic review was challenging. For example, the concept of social media technically falls under the umbrella term mHealth; however, it is its own complex social phenomenon, was recently the subject of an exhaustive review [68], and was therefore considered beyond the scope of this study. Additionally, given the difficulty of achieving the highest quality research in unstable environments, the choice was made to include pre/post studies without formal external controls. As such, the conclusions of this systematic review will need to be validated with future, high quality investigations focusing on evaluation of scaled-up mHealth interventions and the increased use of randomized-controlled trial methodologies. Another limitation of this study is the heterogeneity of outcomes, which precluded a formal meta-analysis. One final weakness is the selection bias introduced by only including publications in English and Spanish.

\section{CONCLUSIONS}

This systematic review found that mHealth is effective in improving provider-focused, process-driven, and patient-centered outcomes in both routine and complex EC settings in LMICs through ensuring the delivery of consistent and high-quality care while improving access to health services. It is an effective means to improve data collection, purvey decision support, provide direct patient care, and offer health trainings in EC in LMICs. Future research with an increased focus on patient-centered outcomes is needed to validate these findings.

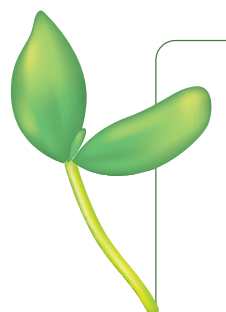

Acknowledgements: We thank the Global Emergency Medicine Literature Review (GEMLR) group and its editorial board for its support and dedication to this research. We also thank the Usher Institute of Population Health Sciences and Informatics for their steadfast support and immense help with this systematic review.

Data sharing: All data supporting the findings of this study are available within the article and its supplementary materials. Otherwise, there are no additional data.

Funding: None.

Authorship contributorship: W. Tyler Winders served as the lead in all phases of the study. Stephanie Garbern, Corey Bills, Pryanka Relan, and Megan Schultz all assisted with study design, protocol optimization, study screening, and manuscript editing. Indi Trehan and Sean Kivlehan served in an advisory role and helped significantly with study design and manuscript development. Torben Becker and Ruth McQuillan served as guarantors and were thus intimately involved in study development, protocol optimization, analysis, synthesis, and manuscript preparation.

Competing interests: All authors have completed the ICMJE Unified Competing Interest form (available on request from the corresponding author) and declare no conflicts of interest.

Additional material

Online Supplementary Document 
1 Kay M, Santos J, Takane M. mHealth: New horizons for health through mobile technologies. Geneva: WHO; 2011.

2 Bastawrous A, Armstrong MJ. Mobile health use in low- and high-income countries: an overview of the peer-reviewed literature. J R Soc Med. 2013;106:130-42. Medline:23564897 doi:10.1177/0141076812472620

3 Finitsis DJ, Pellowski JA, Johnson BT. Text message intervention designs to promote adherence to antiretroviral therapy (ART): a meta-analysis of randomized controlled trials. PLoS One. 2014;9:e88166. Medline:24505411 doi:10.1371/journal. pone.0088166

4 Devi BR, Syed-Abdul S, Kumar A, Iqbal U, Nguyen PA, Li YC, et al. mHealth: An updated systematic review with a focus on HIV/AIDS and tuberculosis long term management using mobile phones. Comput Methods Programs Biomed. 2015;122:25765. Medline:26304621 doi:10.1016/j.cmpb.2015.08.003

5 Gurman TA, Rubin SE, Roess AA. Effectiveness of mHealth behavior change communication interventions in developing countries: a systematic review of the literature. J Health Commun. 2012;17 Suppl 1:82-104. Medline:22548603 doi:10.1080/108 10730.2011 .649160

6 Tang N, Stein J, Hsia RY, Maselli JH, Gonzales R. Trends and characteristics of US emergency department visits, 1997-2007. JAMA. 2010;304:664-70. Medline:20699458 doi:10.1001/jama.2010.1112

7 Obermeyer Z, Abujaber S, Makar M, Stoll S, Kayden SR, Wallis LA, et al. Emergency care in 59 low-and middle-income countries: a systematic review. Bull World Health Organ. 2015;93:577-86. Medline:26478615 doi:10.2471/BLT.14.148338

8 Heudtlass P, Speybroeck N, Guha-Sapir D. Excess mortality in refugees, internally displaced persons and resident populations in complex humanitarian emergencies (1998-2012) - insights from operational data. Confl Health. 2016;10:15. Medline:27441038 doi:10.1186/s13031-016-0082-9

9 Covidence systematic review software. Melbourne, Australia: Veritas Health Innovation; 2020.

10 Moorhead SA, Hazlett DE, Harrison L, Carroll JK, Irwin A, Hoving C. A new dimension of health care: systematic review of the uses, benefits, and limitations of social media for health communication. J Med Internet Res. 2013;15:e85. Medline:23615206 doi:10.2196/jmir. 1933

11 Guyatt G, Oxman AD, Akl EA, Kunz R, Vist G, Brozek J, et al. GRADE guidelines: 1. Introduction-GRADE evidence profiles and summary of findings tables. J Clin Epidemiol. 2011;64:383-94. Medline:21195583 doi:10.1016/j.jclinepi.2010.04.026

12 Moher D, Shamseer L, Clarke M, Ghersi D, Liberati A, Petticrew M, et al. Preferred reporting items for systematic review and meta-analysis protocols (PRISMA-P) 2015 statement. Syst Rev. 2015;4:1. Medline:25554246 doi:10.1186/2046-4053-4-1

13 World Health Organization. Definition of regional groupings. 2020. Available: https://www.who.int/healthinfo/global_burden_disease/definition_regions/en/\#: :text=WHO\%20regions\%3A\%20WHO\%20Member\%20States,Region\%2C\%20and\%20 Western\%20Pacific\%20Region. Accessed 17 March 2020.

14 Finette BA, McLaughlin M, Scarpino SV, Canning J, Grunauer M, Teran E, et al. Development and Initial Validation of a Frontline Health Worker mHealth Assessment Platform (MEDSINC (R)) for Children 2-60 Months of Age. Am J Trop Med Hyg. 2019;100:1556-65. Medline:30994099 doi:10.4269/ajtmh.18-0869

15 Bengtsson L, Gaudart J, Lu X, Moore S, Wetter E, Sallah K, et al. Using Mobile Phone Data to Predict the Spatial Spread of Cholera. Sci Rep. 2015;5:8923. Medline:25747871 doi:10.1038/srep08923

16 Bengtsson L, Lu X, Thorson A, Garfield R, von Schreeb J. Improved response to disasters and outbreaks by tracking population movements with mobile phone network data: a post-earthquake geospatial study in Haiti. PLoS Med. 2011;8:e1001083. Medline:21918643 doi:10.1371/journal.pmed.1001083

17 Davies C, Graffy R, Shandukani M, Baloyi E, Gast L, Kok G, et al. Effectiveness of 24-h mobile reporting tool during a malaria outbreak in Mpumalanga Province, South Africa. Malar J. 2019;18:45. Medline:30791909 doi:10.1186/s12936-019-2683-4

18 El-Khatib Z, Shah M, Zallappa SN, Nabeth P, Guerra J, Manengu CT, et al. SMS-based smartphone application for disease surveillance has doubled completeness and timeliness in a limited-resource setting - evaluation of a 15-week pilot program in Central African Republic (CAR). Confl Health. 2018;24:12-42. Medline:30386418 doi:10.1186/s13031-018-0177-6

19 Feng S, Grepin KA, Chunara R. Tracking health seeking behavior during an Ebola outbreak via mobile phones and SMS. NPJ Digit Med. 2018;1:51. Medline:31304330 doi:10.1038/s41746-018-0055-z

20 Jia KB, Mohamed K. Evaluating the use of cell phone messaging for community Ebola syndromic surveillance in high risked settings in Southern Sierra Leone. Afr Health Sci. 2015;15:797-802. Medline:26957967 doi:10.4314/ahs.v15i3.13

21 Toda M, Njeru I, Zurovac D, S O-Tipo, Kareko D, Mwau M, et al. The impact of a SMS-based disease outbreak alert system (mSOS) in Kenya. Int J Inf Dis. 2016;455:247. doi:10.1016/j.ijid.2016.02.553

22 Yugi J, Buesseler HM. Text messaging app improves disease surveillance in rural South Sudan. mHealth. 2016;2:8. Medline:28293586 doi:10.21037/mhealth.2016.03.01

23 Amoakoh HB, Klipstein-Grobusch K, Grobbee DE, Amoakoh-Coleman M, Oduro-Mensah E, Sarpong C, et al. Using Mobile Health to Support Clinical Decision-Making to Improve Maternal and Neonatal Health Outcomes in Ghana: Insights of Frontline Health Worker Information Needs. JMIR Mhealth Uhealth. 2019;7:e12879. Medline:31127719 doi:10.2196/12879

24 Bavdekar SB, Pawar M. Evaluation of an Internet delivered pediatric diagnosis support system (ISABEL) in a tertiary care center in India. Indian Pediatr. 2005;42:1086-91. Medline:16340049

25 Blom L, Boissin C, Allorto N, Wallis L, Hasselberg M, Laflamme L. Accuracy of acute burns diagnosis made using smartphones and tablets: a questionnaire-based study among medical experts. BMC Emerg Med. 2017;17:39. Medline:29237400 doi:10.1186/s12873-017-0151-4

26 Crehan C, Kesler E, Nambiar B, Dube Q, Lufesi N, Giaccone M, et al. The NeoTree application: developing an integrated mHealth solution to improve quality of newborn care and survival in a district hospital in Malawi. BMJ Glob Health. 2019;4:e000860. Medline:30713745 doi:10.1136/bmjgh-2018-000860 
27 Duffy S, MacLaren E, Phillips D, Letchworth P. MedNav - helping midwives manage neonatal resuscitation in resource poor settings. Future Healthc J. 2017;4:178-83. Medline:31098467 doi:10.7861/futurehosp.4-3-178

28 Haque F, Ball RL, Khatun S, Ahmed M, Kache S, Chisti MJ, et al. Evaluation of a Smartphone Decision-Support Tool for Diarrheal Disease Management in a Resource-Limited Setting. PLoS Negl Trop Dis. 2017;11:e0005290. Medline:28103233 doi:10.1371/journal.pntd.0005290

29 Savatmongkorngul S, Yuksen C, Suwattanasilp C, Sawanyawisuth K, Sittichanbuncha Y. Is a mobile emergency severity index (ESI) triage better than the paper ESI? Intern Emerg Med. 2017;12:1273-7. Medline:27878444 doi:10.1007/s11739-016-1572-x

30 Bilal S, Nelson E, Meisner L, Alam M, Al Amin S, Ashenafi Y, et al. Evaluation of Standard and Mobile Health-Supported Clinical Diagnostic Tools for Assessing Dehydration in Patients with Diarrhea in Rural Bangladesh. Am J Trop Med Hyg. 2018;99:1719. Medline:29761756 doi:10.4269/ajtmh.17-0648

31 Brangel P, Sobarzo A, Parolo C, Miller BS, Howes PD, Gelkop S, et al. A Serological Point-of-Care Test for the Detection of IgG Antibodies against Ebola Virus in Human Survivors. ACS Nano. 2018;12:63-73. Medline:29303554 doi:10.1021/acsnano. $7 \mathrm{~b} 07021$

32 Chauhan V, Negi PC, Raina S, Raina S, Bhatnagar M, Guleri R, et al. Smartphone-based tele-electrocardiography support for primary care physicians reduces the pain-to-treatment time in acute coronary syndrome. J Telemed Telecare. 2018;24:540-6. Medline:28750576 doi:10.1177/1357633X17719395

33 Chen H, Liu J, Xiang D, Qin W, Zhou M, Tian Y, et al. Coordinated digital-assisted program improved door-to-balloon time for acute chest pain patients. Int Heart J. 2016;57:310-6. Medline:27150005 doi:10.1536/ihj.15-415

34 Chinprasatsak S, Satthapong S, Kotruchin P, Maporn K. Telegraphic medicine systems improve medical diagnosis in pre-hospital settings: A pilot study in a tertiary care hospital. J Med Assoc Thai. 2017;6:686-91.

35 Dharmasaroja PA, Muengtaweepongsa S, Kommarkg U. Implementation of Telemedicine and Stroke Network in Thrombolytic Administration: Comparison Between Walk-in and Referred Patients. Neurocrit Care. 2010;13:62-6. Medline:20411354 doi:10.1007/s12028-010-9360-3

36 Filho GSF, Solla DJF, Argolo FC, Guimaraes PO, Filho IMP, Carvalho LGM, et al. Implementation of a regional network for st-segment-elevation myocardial infarction (STEMI) care and 30-day mortality in a low- to middle-income city in Brazil: Findings from Salvador's stemi registry (RESISST). J Am Heart Assoc. 2018;7:e008624. Medline:29980522

37 Garde A, Zhou GH, Raihana S, Dunsmuir D, Karlen W, Dekhordi P, et al. Respiratory rate and pulse oximetry derived information as predictors of hospital admission in young children in Bangladesh: a prospective observational study. BMJ Open. 2016;6:e011094. Medline:27534987 doi:10.1136/bmjopen-2016-011094

38 Ma C, Gunaratnam LC, Ericson A, Conroy AL, Namasopo S, Opoka RO, et al. Handheld Point-of-Care Lactate Measurement at Admission Predicts Mortality in Ugandan Children Hospitalized with Pneumonia: A Prospective Cohort Study. Am J Trop Med Hyg. 2019;100:37-42. Medline:30398141 doi:10.4269/ajtmh.18-0344

39 Macedo TA, de Barros ESPG, Simoes SA, Okada MY, Garcia JC, Sampaio MC, et al. Impact of Chest Pain Protocol with Access to Telemedicine on Implementation of Pharmacoinvasive Strategy in a Private Hospital Network. Telemed J E Health. 2016;22:549-52. Medline:26693879 doi:10.1089/tmj.2015.0178

40 Marcolino MS, Brant LC, Araujo JG, Nascimento BR, Castro LR, Martins P, et al. Implementation of the myocardial infarction system of care in city of Belo Horizonte, Brazil. Arq Bras Cardiol. 2013;100:307-14. Medline:23545995 doi:10.5935/abc.20130054

41 Rahman A, Akhter S, Nisha MK, Islam SS, Ashraf F, Rahman M, et al. Can mHealth improve access to safe blood for transfusion during obstetric emergency? Int J Womens Health. 2017:9:235-43. Medline:28461767 doi:10.2147/IJWH.S120157

42 Saberian P, Tavakoli N, Ramim T, Hasani-Sharamin P, Shams E, Baratloo A. The Role of Pre-Hospital Telecardiology in Reducing the Coronary Reperfusion Time; a Brief Report. Arch Acad Emerg Med. 2019;7:e15. Medline:30847450

43 Steinhubl SR, Feye D, Levine AC, Conkright C, Wegerich SW, Conkright G. Validation of a portable, deployable system for continuous vital sign monitoring using a multiparametric wearable sensor and personalised analytics in an Ebola treatment centre. BMJ Glob Health. 2016;1:e000070. Medline:28588930 doi:10.1136/bmjgh-2016-000070

44 Tirivayi NM, Tomini P, Tesfaye S, Chikulo W, Morelli C. Humanitarian Assistance through Mobile Cash Transfers. United Nations University. 2016:1-98.

45 Zachariah R, Bienvenue B, Ayada L, Manzi M, Maalim A, Engy E, et al. Practicing medicine without borders: tele-consultations and tele-mentoring for improving paediatric care in a conflict setting in Somalia? Trop Med Int Health. 2012;17:115662. Medline:22845678 doi:10.1111/j.1365-3156.2012.03047.x

46 Bolan NE, Sthreshley L, Ngoy B, Ledy F, Ntayingi M, Makasy D, et al. mLearning in the Democratic Republic of the Congo: A Mixed-Methods Feasibility and Pilot Cluster Randomized Trial Using the Safe Delivery App. Glob Health Sci Pract. 2018;6:693710. Medline:30591577 doi:10.9745/GHSP-D-18-00275

47 Carrillo-Larco RM, Shu-Yip SB, Perez-Lu JE. Academic application of text messages in a first aid course: a pilot study in a private university in Lima, Peru. Rev Peru Med Exp Salud Publica. 2015;32:278-82. Medline:26338387 doi:10.17843/rpmesp.2015.322.1620

48 Castro LA, Favela J, Garcia-Pena C. Effects of communication media choice on the quality and efficacy of emergency calls assisted by a mobile nursing protocol tool. Comp Inform Nurs. 2014;32:550-8. Medline:25251859 doi:10.1097/ CIN.0000000000000101

49 Jain A, Agarwal R, Chawla D, Paul V, Deorari A. Tele-education vs classroom training of neonatal resuscitation: a randomized trial. J Perinatol. 2010;30:773-9. Medline:20357810 doi:10.1038/jp.2010.42

50 Kovacevic P, Dragic S, Kovacevic T, Momcicevic D, Festic E, Kashyap R, et al. Impact of weekly case-based tele-education on quality of care in a limited resource medical intensive care unit. Crit Care. 2019;23:220. Medline:31200761 doi:10.1186/ s13054-019-2494-6 
51 Lin M, Brooks TN, Miller AC, Sharp JL, Hai LT, Nguyen T, et al. Impact of an English-based pediatric software on physician decision making: A multicenter study in Vietnam. West J Med. 2013;S176-7.

52 Liu X, Cheng J, Huang S. Mobile phone training platform for the nursing staff in the emergency department. Telemed J E Health. 2019;25:66-70. Medline:29742034 doi:10.1089/tmj.2017.0317

53 Lund S, Boas IM, Bedesa T, Fekede W, Nielsen HS, Sorensen BL. Association Between the Safe Delivery App and Quality of Care and Perinatal Survival in Ethiopia A Randomized Clinical Trial. JAMA Pediatr. 2016;170:765-71. Medline:27322089 doi:10.1001/jamapediatrics.2016.0687

54 Mikrogianakis A, Kam A, Silver S, Bakanisi B, Henao O, Okrainec A, et al. Telesimulation: an innovative and effective tool for teaching novel intraosseous insertion techniques in developing countries. Acad Emerg Med. 2011;18:420-7. Medline:21496146 doi:10.1111/j.1553-2712.2011.01038.x

55 Onan A, Turan S, Elcin M, Erbil B, Bulut SC. The effectiveness of traditional Basic Life Support training and alternative technology-enhanced methods in high schools. Hong Kong J Emerg Med. 2019;26:44-52. doi:10.1177/1024907918782239

56 Otu A, Ebenso B, Okuzu O, Osifo-Dawodu E. Using a mHealth tutorial application to change knowledge and attitude of frontline health workers to Ebola virus disease in Nigeria: a before-and-after study. Hum Resour Health. 2016;14:5. Medline:26872824 doi:10.1186/s12960-016-0100-4

57 Saberi F, Adib-Hajbaghery M, Zohrehie J. The effects of public education through Short Message Service on the time from symptom onset to hospital arrival in patients with myocardial infarction: A field trial. ARYA Atheroscler. 2017;13:97-102. Medline:29147118

58 Terry B, Polan DL, Nambaziira R, Mugisha J, Bisanzo M, Gaspari R. Rapid, remote education for point-of-care ultrasound among non-physician emergency care providers in a resource limited setting. Afr J Emerg Med. 2019;9:140-4. Medline:31528532 doi:10.1016/j.afjem.2019.05.004

59 Yigzaw M, Tebekaw Y, Kim YM, Kols A, Ayalew F, Eyassu G. Comparing the effectiveness of a blended learning approach with a conventional learning approach for basic emergency obstetric and newborn care training in Ethiopia. Midwifery. 2019;78:429. Medline:31349183 doi:10.1016/j.midw.2019.07.014

60 Park J, Choi KH, Lee JM, Kim HK, Hwang D, Rhee TM, et al. Prognostic Implications of Door-to-Balloon Time and Onset-toDoor Time on Mortality in Patients With ST -Segment-Elevation Myocardial Infarction Treated With Primary Percutaneous Coronary Intervention. J Am Heart Assoc. 2019;8:e012188. Medline:31041869 doi:10.1161/JAHA.119.012188

61 Dharmasaroja PA, Muengtaweepongsa S, Kommarkg U. Implementation of Telemedicine and Stroke Network in thrombolytic administration: comparison between walk-in and referred patients. Neurocrit Care. 2010;13:62-6. Medline:20411354 doi:10.1007/s12028-010-9360-3

62 Acharibasam JW, Wynn R. Telemental Health in Low- and Middle-Income Countries: A Systematic Review. Int J Telemed Appl. 2018;2018:9602821. Medline:30519259 doi:10.1155/2018/9602821

63 Marcolino MS, Oliveira JAQ, D’Agostino M, Ribeiro AL, Alkmim MBM, Novillo-Ortiz D. The impact of mHealth interventions: systematic review of systematic reviews. JMIR Mhealth Uhealth. 2018;6:e23. Medline:29343463 doi:10.2196/mhealth.8873

64 White A, Thomas DSK, Ezeanochie N, Bull S. Health Worker mHealth Utilization: A Systematic Review. Computers, informatics, nursing. CIN. 2016;34:206-13. Medline:26955009

65 Jollis JG, Roettig ML, Aluko AO, Anstrom KJ, Applegate RJ, Babb JD, et al. Implementation of a statewide system for coronary reperfusion for ST-segment elevation myocardial infarction. JAMA. 2007;298:2371-80. Medline:17982184 doi:10.1001/ jama.298.20.joc70124

66 Zhang D, Shi L, Ido MS, Green DE, Li Y, Su D, et al. Impact of Participation in a Telestroke Network on Clinical Outcomes. Circ Cardiovasc Qual Outcomes. 2019;12:e005147. Medline:30606053 doi:10.1161/CIRCOUTCOMES.118.005147

67 Steinhubl SR, Feye D, Levine AC, Conkright C, Wegerich SW, Conkright G. Validation of a portable, deployable system for continuous vital sign monitoring using a multiparametric wearable sensor and personalised analytics in an Ebola treatment centre. BMJ Glob Health. 2016;1:e000070. Medline:28588930 doi:10.1136/bmjgh-2016-000070

68 Walker T, Quintanilla J, Stoll P. Humanitarian Futures for Messaging Apps: Understading the opportunities and risks for humanitarian action. International Committee of the Red Cross, The Engine Room, The Block Party: 2017. 